\title{
DOES MOBILITY INCREASE THE PRODUCTIVITY OF INVENTORS?
}

\author{
KARIN HOISL
}

Discussion Paper 2006-13

August - 2007

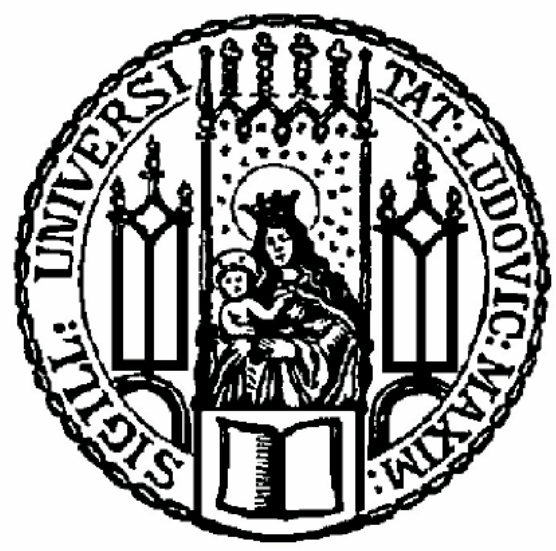

MUNiCH SCHOOL OF MANAGEMENT UNIVERSITY OF MUNICH

FAKULTÄT FÜR BETRIEBSWIRTSCHAFT LUDWIG-MAXIMILIANS-UNIVERSITÄT MÜNCHEN

Online at http://epub.ub.uni-muenchen.de/ 


\title{
Does Mobility Increase the Productivity of Inventors?
}

\author{
Karin Hoisl \\ Institute for Innovation Research, Technology Management and Entrepreneurship, University of Munich, \\ Kaulbachstraße 45, D-80539 Munich, Germany \\ Tel.: +49-89-2180-5626; Fax: +49-89-2180-6284; email: hoisl@bwl.uni-muenchen.de
}

\begin{abstract}
:
Although labor mobility has been recognized as a key mechanism for transferring tacit knowledge, prior research on inventors has so far hardly discussed the impact of a move on inventive performance. Additionally, existing research has neglected the differences in gains from a move between high and lower performing inventors. This paper adds to the current R\&D literature by presenting a jointly estimated quantile regression to compare the coefficients of the explanatory variables at different points of the performance distribution. Additionally, dummy variables are used to compare inventive performance prior and in the aftermath of a move. Results reveal that inventors at the upper end of the performance distribution are better able to benefit from a move to draw level with or to overtake nonmovers in the post-move period. Whereas at the bottom of the performance distribution a higher level of education has a positive impact on inventive performance, education does not matter significantly at the upper end of the performance distribution. Data for the analysis was derived from a survey of German inventors $(\mathrm{N}=3,049)$.
\end{abstract}

Keywords: Inventor, Mobility, Quantile Regression, Patent

\section{Acknowledgements}

I would like to thank the conference audience at the 33rd Conference of E.A.R.I.E. in August 2006, the conference audience at the $1^{\text {st }}$ Annual Conference of the EPIP Association "Policy, Law and Economics of Intellectual Property" in September 2006, and the audience at the DRUID Summer Conference in June 2007 for helpful comments. Special thanks go to Dietmar Harhoff, Jesse Giummo, Marc Gruber, Pierre Mohnen, Mark Schankerman and an anonymous referee for their valuable comments. The survey responses used in this analysis originate from a coordinated survey effort in Italy, France, Spain, the Netherlands, the United Kingdom and Germany. The author thanks the European Commission, Contract N. HPV2-CT-2001-00013, for supporting the creation of the joint dataset. This paper makes use of the German survey responses. The usual caveat applies. 


\section{$1 \quad$ Introduction}

“We know more than we can tell" (Polanyi 1958, p. 55)

Especially in the context of tacit knowledge, this oft-quoted statement of Polanyi must be accepted to be true. Since knowledge is one of the most important inputs into innovative activity, an often-discussed phenomenon in the literature is the access to knowledge after hiring a key inventor from another firm. Researchers argue that a move ${ }^{1}$ of an inventor to another firm can lead to knowledge transfers (Arrow 1962; Almeida/Kogut 1999; Moen 2000; Rosenkopf/Almeida 2001; Agrawal et al. 2003). Firms characterized by a "lower technology" level can use this knowledge to catch up and thus are motivated to attract productive inventors (Gilfillan 1935; Song et al. 2003). In particular, the transfer of tacit knowledge that is otherwise immobile is facilitated by inventor mobility (Dosi 1988).

What has so far hardly been addressed in the inventor related literature is the impact of a move on inventive performance. Trajtenberg $(2005,2006)$ is one of the first to analyze the relationship between mobility and labor productivity for R\&D personnel. He uses data on 1,565,780 inventors listed on U.S. patent documents and finds that mobility has a positive impact on work performance; in particular, patents of mobile inventors receive more citations. However, Trajtenberg (2005) does not differentiate between the pre-move and the post-move period. Using instrumental variables techniques, Hoisl (2007a) shows that there exists a simultaneous relationship between inventor mobility and inventor productivity: movers are more productive than non-moving inventors. Moreover, more productive inventors are less likely to move. These results confirm the findings of Topel and Ward (1992) who propose that mobility can lead to an improvement of the match between employer and employee. A

\footnotetext{
1 In the following study, a move of an inventor is defined as a change of employer.
} 
better match quality should then lead to an increase in the inventor's productivity. This view implies that inventors with poor matches move and that inventors who have already found a good match do not change their employer. Schankerman et al. (2006) analyze the impact of the performance of more than 50,000 software inventors on the probability of movement. The authors do not find evidence that matching is an important incentive for movement in the software industry. In fact, asymmetric information between employer and employee about the value of an invention turned out to be a relevant incentive for a move. In particular, ex-ante ${ }^{2}$ observable indicators (e.g., the number of claims) decrease the probability to observe a move. Information, which is only available ex-post (e.g., the number of citations a patent receives) has a positive impact on movement (Schankerman et al. 2006).

However, the existing literature does not allow interpreting the increase in productivity with respect to a particular move, since previous studies have not distinguished between the performance of an inventor in the pre- and in the post-move period. To improve on the current literature, this study employs a treatment (movers) and a control group (non-movers) to differentiate between the inventive output of movers and non-movers. Dummy variables will be factored into the regression to compare inventive performance in the pre- and the postmove period. Additionally, a jointly estimated quantile regression is used to compare the coefficients of the explanatory variables describing the $0.25,0.5$ and 0.75 quantile of inventive performance. This approach allows for analyzing differences in gains from movement between high and lower performing inventors.

This paper makes use of data collected in a large-scale survey of German inventors who hold at least one granted European patent. The data include demographic information on more than 3,000 inventors, for instance, the age and the educational degree that will be used to construct

2 Ex-ante means at the time of the patent application. 
an appropriate control group. Patent data from the online EPOLINE database of the European Patent Office (EPO) was further used to trace mobility of inventors over time. All patent applications of the surveyed inventors with priority years between 1985 and 1999 were included.

Results reveal that a move has a positive impact on inventive performance. In particular, movers are able to catch up with or to overtake the non-movers in the after-move period. These results confirm the importance of match quality for inventive performance. Results further reveal that inventors at the upper end of the performance distribution are better able to benefit from a move. Additionally, whereas inventors at the bottom of the performance distribution profit from a higher level of education, inventors at the upper end of the distribution benefit from the allocation of resources.

The remainder of this paper is organized as follows. Section 2 contains a description of the dataset and of the variables employed in the empirical analysis. Section 3 provides a characterization of the methodology used and describes the construction of the control group. Section 5 contains descriptive and multivariate results. Finally, section 6 presents a discussion of the outcomes and provides implications for further research.

\section{Data Source and Sample}

\subsection{Description of the Data}

The data used in this study were collected in the course of a project, sponsored by the European Commission, called $\mathrm{PatVal}^{3}$. Research groups from six European universities participated in this project. In each of the six countries (France, Germany, Great Britain, Italy, Spain, and the Netherlands) domestic inventors were surveyed simultaneously regarding their

3 PatVal is the acronym for "The Value of European Patents: Empirical Models and Policy Implications Based on a Survey of European Inventors". 
granted EP patents. ${ }^{4}$ The following analysis relies on the German data. 10,500 EP patents of inventors who lived in Germany at the time of application were chosen by stratified random sampling. ${ }^{5}$ The sampling procedure was based on a list of all granted EP patents with priority dates between 1993 and 1997 (15,595 EP patents). A stratified random sample was used in order to oversample potentially important patents.

The information was obtained using a questionnaire. The first inventor listed on the patent document was chosen as the addressee of the survey; 3,049 responses were received. The data from the questionnaire were merged with bibliographic and procedural information on the respective patents, obtained from the online EPOLINE database. The database contains information on all published EP patent applications as well as all published PCT applications since the founding of the EPO in 1978. The dataset corresponds to the EPOLINE data as of March 1st, 2003. To trace the mobility of each inventor over time, the EPOLINE database was further used to search for all patent applications belonging to the 3,049 inventors with priority dates between 1985 and $1999^{6}$. Inventors holding only one patent (352 inventors) were removed from the sample since a move is only observable if the inventor is responsible for at least two patent applications during the time period under consideration. Therefore, the final sample contains 2,697 inventors.

4 See Giuri, Mariani et al. (2005) for a detailed description of the PatVal-EU survey results.

5 The sample of 10,500 patents includes all opposed patents $(1,048)$ and patents which were not opposed but received at least one citation (5,333), and a random sample of 4,119 patents drawn from the remaining 9,212 patents.

6 The lower limit (1985) was chosen, since the years between 1977 and 1984 are characterized by a strong increase in the number of European patent applications, which was caused by the diffusion of the European patent after the founding of the EPO in 1978. Hence, I assume that as of mid-1980, European patent data are a sufficiently reliable source of data to use for an empirical analysis. The upper limit (1999) was chosen due to the limitations in the availability of citation data. To count the number of citations a patent received from subsequent patents and to compare citations between patents applied for in different years, the number of citations received within a four year time lag from the publication of the search report was employed. Since the search report is published about one year after a firm or an individual inventor applied for the EP patent, patent data as of 2004 are needed to calculate four years citation lags for patents with priority year 1999. 
Before describing the research methodology, a few limitations of patent data to trace mobility need to be mentioned. First, a lack of standardization of the spelling of inventors' names in the patent documents leads to a name matching problem. This matching problem complicates the search for all patent applications per inventor, especially for inventors with common last names. Identical names may refer to different inventors and different spellings may refer to the same inventor. Second, incomplete address data and the fact that some female inventors changed their names due to marriage lead to wrong matches or failure to match. Even if the matching procedure works well, it is only possible to identify a move if the inventor applied for another patent after he changed the employer. This may lead to a selection bias, since the probability to observe a move increases with the number of patents per inventor, i.e. the probability to observe a move is higher for productive inventors. Furthermore, the fact that different applicants are listed on a patent document does not automatically mean that the inventor changed jobs. A possible explanation for two different applicants is, for instance, a strategic alliance between two companies or a merger. To reduce biases, the classification of "move" (the inventor changed the employer) and "no move" (the inventor did not change the employer) was corrected manually after intensive searches. ${ }^{7}$ Additionally, the results from the PatVal questionnaire, including responses related to the mobility of the inventors, were utilized to confirm the matching and mobility outcomes. Despite these efforts, the resulting data continue to have some limitations and one ought to be cautious when deriving implications from the results.

\footnotetext{
7 For more information about the name matching procedure, see Hoisl (2007a).
} 


\subsection{Description of the Variables}

A brief description of the variables to be used as well as their role in the following multivariate analysis will be given in the next paragraphs.

Age - The variable contains the age of the inventors in 1999. The variable will be used to construct the control group and will also be a control variable in the regression to capture the experience of inventors.

Level of education - The questionnaire included a question asking the respondents for their terminal degree. The education variable was aggregated to three groups: (1) secondary school, high school diploma, or vocational training, (2) vocational academy (Berufsakademie) or university studies, and (3) doctoral or postdoctoral studies. Again this variable will be used to construct the control group and will also be factored into the regression as a proxy for the ability of inventors.

Technical field - Based on their International Patent Classification (IPC) codes, the patent applications were classified into 6 main technical areas. This classification was proposed by Schmoch (OECD 1994). The main technical areas were used as a third criteria to match inventors into the control group.

Number of citations per patent - This variable includes the average number of citations the patent applications of an inventor received within 4 years following the publication of the search reports. According to Harhoff et al. (2003) the number of citations is a proxy for the value of a patent. Therefore, this variable will be used as a measure for output quality.

Multiple movement - The information whether an inventor moved (= changed his employer) or not will again be used to construct the control group. In case an inventor moved repeatedly within the time period under consideration, one of the moves was selected at random. To control for multiple movement in the regression, a dummy variable was constructed, taking 
the value 1 for inventors who moved more than once during their "inventive life" (i.e. between 1985 and 1999), and 0 otherwise.

Inventor team size - This variable provides information about the size of the inventor team, i.e., it contains the number of inventors mentioned on the patent document. Team size will be included in the regression to control for the allocation of resources in different R\&D projects and also for firm size.

Technical concentration - Using 30 technical areas (OECD 1994), a Herfindahl index was calculated. For each inventor, the number of applications in the technical area $i$ divided by the total number of applications was calculated, in the following denoted by $p$. The Herfindahl index (HI) corresponds to the sum of squared shares of applications:

$$
H I=\sum_{i} p_{i}^{2}
$$

If all patent applications of an inventor belong to one technical area, technical concentration is at its maximum and the Herfindahl index is equal to 1.

\section{$3 \quad$ Research Methodology}

To compare the performance of movers and non-movers in the pre- and post-move period, a pre-post design with control group will be employed. First, movers were assigned to the treatment group. These inventors had to be at least 25 years old in 1986, since it is assumed that inventors do not become active before the age of 25 . Additionally, treatment inventors had to have changed their employer at least once between 1990 and 1995. Subsequently, a non-moving control inventor was assigned to each inventor of the treatment group using a matching approach. In particular, three matching criteria were employed: the age of the inventor, his educational background as well as the main technical area in which the inventor is active. Additionally, a congruent 4 year time window before and after the move was chosen, since different time periods could have resulted in biases due to a different patent 
behavior at different points in time (Hall 2004). In case two or more inventors were potential candidates for matched pairs, one of these inventors was selected at random. Overall, matched pairs could be found for 352 mobile inventors, resulting in a dataset of 704 inventors who have been responsible for a total of 11,273 patent applications between 1985 and 1999. To measure the performance of the inventors, the average number of citations per patent will be used.

Since a pre-post design requires knowledge of the specific point in time when the move took place, information on the applicants of the patents and the priority dates were used to calculate a proxy for the exact date of the move. To identify whether a move actually occurred, applicant names listed on the EP patent documents were employed. In the event that two successive patent documents belonging to the same inventor contained two different applicants, it was assumed that the inventor changed his employer in the time period between these two patent applications. Since the exact time of move was not available, the move date was estimated by taking the midpoint between the two application dates (the last patent before and the first patent after the move). In case inventors changed their employer more than once between 1990 and 1995, one of these moves was selected at random.

In the following, an OLS regression framework will be employed. In particular, equation (2) will be estimated:

$$
\begin{aligned}
y & =\beta_{0}+\beta_{1} * \text { mobile_pre }+\beta_{2} * \text { mobile_post }+\beta_{3} * \text { stay _ pre } \\
& +\beta_{4 k} * \text { control_variables }{ }_{k}+u
\end{aligned}
$$

where mobile_pre is a dummy variable, taking the value one for movers in the pre-move period. mobile_post is a dummy variable, taking the value one for movers in the post-move period. Finally, the dummy stay_pre becomes one for non-movers in the pre-move period. stay_post is used as a reference group and refers to non-movers in the post-move period. The 
control_variables include characteristics of the inventor, e.g., the age or the level of education and characteristics of the employer, e.g., the average size of the inventor teams.

To learn more about the gains from moving, a quantile regression will be employed. A quantile regression can be defined as a regression based method to model the quantiles of the dependent variable, conditional on the explanatory variables (Koenker/Bassett 1978). In the way that OLS estimates the impact of the explanatory variables at the conditional mean of the dependent variable, quantile regression allows to estimate the impact of the explanatory variables at different points of the conditional distribution, e.g., the 0.25 quantile or the median (Eide/Showalter 1999). In economics, and especially in labor economics, a "rapidly expanding empirical quantile regression literature" can be observed (Koenker/Hallock 2001: 151). ${ }^{8}$ In the following the quantile-regression model according to Koenker and Bassett (1978) is estimated:

$$
\begin{gathered}
Q_{\theta}(y \mid x)=\gamma_{\theta, 0}+\gamma_{\theta, 1} * \text { mobile_pre }+\gamma_{\theta, 2} * \text { mobile_post }+\gamma_{\theta, 3} * \text { stay_pre } \\
+\gamma_{\theta, 4 k} * \text { control_variables }_{k}
\end{gathered}
$$

where $Q_{\theta}(y \mid x)$ denotes the $\theta$ th conditional quantile of $\mathrm{y}$ given $\mathrm{x}$. The model will be estimated by the $0.25,0.5$ and 0.75 quantiles.

To calculate and to test differences between coefficients describing the three quantiles, a jointly estimated quantile regression is used. The resulting coefficients will be the same as those for estimating each equation separately, using a quantile regression approach. The difference between both methods is that the joint approach produces an estimate of the variance-covariance matrix of the quantile estimators via bootstrapping ${ }^{9}$, which allows testing differences in the coefficients describing the considered quantiles.

\footnotetext{
8 See, e.g., Chamberlain (1994), Buchinsky (1994) and Fitzenberger (1999) for an application of the quantile regression method in empirical studies comprising labor market related issues.

9 For a more detailed description of the bootstrap percentile method, see Hahn (1995).
} 


\section{Results}

\subsection{Descriptive Results}

Table 1 provides descriptive statistics of the above specified variables for the treatment and the control group separately. As already mentioned, the final sample contains 352 mobile inventors and 352 non-mobile control inventors, who have the same age, educational background and technical specialization.

Table 1

Descriptive statistics of the treatment group $\left(\mathrm{N}_{\text {treat }}=352\right)$ and the control group $\left(\mathrm{N}_{\text {control }}=352\right)$; pre/post variables refer to the 4 year period

\begin{tabular}{lrrrrrrrc}
\hline & \multicolumn{3}{c}{ Mobile inventors $(\mathbf{N}=352)$} & \multicolumn{3}{c}{ Control inventors $(\mathbf{N}=\mathbf{3 5 2})$} \\
\multicolumn{1}{c}{ Variable } & Mean & S.D. & Min. & Max. & Mean & S.D. & Min. & Max. \\
\hline cumulative number of citations * & 19.56 & 32.26 & 0 & 372 & 22.16 & 40.80 & 0 & 547 \\
$\begin{array}{l}\text { number of citations per patent application } \\
\text { multiple movement }\end{array}$ & 3.37 & 2.74 & 0 & 20 & 3.30 & 2.37 & 0 & 13.93 \\
$\begin{array}{l}\text { size of the inventor team ** } \\
\text { secondary school/vocational training/ }\end{array}$ & 0.55 & 0.50 & 0 & 1 & 0 & 0 & 0 & 0 \\
$\quad 3.09$ & 1.37 & 1 & 9 & 3.25 & 1.48 & 1 & 8 \\
$\quad$ high school diploma & 0.09 & 0.29 & 0 & 1 & 0.09 & 0.29 & 0 & 1 \\
$\quad$ university studies & 0.43 & 0.50 & 0 & 1 & 0.43 & 0.50 & 0 & 1 \\
$\quad$ doctoral/post-doctoral studies & 0.48 & 0.50 & 0 & 1 & 0.48 & 0.50 & 0 & 1 \\
technical concentration & 0.77 & 0.23 & 0 & 1 & 0.78 & 0.23 & 0 & 1 \\
age in 2003 & 55.02 & 8.64 & 42 & 74 & 55.02 & 8.64 & 42 & 74 \\
\hline \hline
\end{tabular}

* In a one-sided t-test, the difference between movers and non-movers turned out to be significant.

** In a two-sided t-test, the difference between movers and non-movers turned out to be significant.

The patent applications of mobile inventors received an average of 19.56 citations from subsequent patents (each application received on average 3.37 citations). Non-mobile inventors received on average 22.16 citations (3.30 citations per patent application). The difference between the two groups is significant at the $10 \%$ level. Although the average of the total number of citations is higher for the non-movers, the movers overtake their control inventors in case the number of citations per patent application is considered. This difference 
can be explained by the fact that the non-movers not only receive more (cumulative) citations but also hold more patents.

About $55 \%$ of the mobile inventors moved repeatedly (28\% of the overall sample). Furthermore, the inventors (movers and non-movers) are characterized by a high level of education. More than $90 \%$ of the inventors earned a university degree. $48 \%$ even have a doctoral degree. The age of the inventors varies between 42 and 74 years and has its mean at 55 years. Overall, inventors are rather specialized, the technical concentration amounts to $77 \%$ for movers and to $78 \%$ for non-movers. Furthermore, mobile inventors work in teams that consist of an average of 3 inventors, varying between 1 and 9 (maximum team size for non-movers $=8$, mean $=3$ ). The difference between movers and non-movers as to inventor team size is significant at the $5 \%$ level.

\subsection{Multivariate Results}

Table 2 compares the results of an OLS and a jointly estimated quantile regression analysis. Models 1 and 2 refer to the OLS results. Model 2 additionally includes a control dummy for multiple movement. Models 3 and 4 contain the outcomes of the jointly estimated quantile regression at the $0.25,0.5$ and 0.75 quantiles. Again, Model 4 includes an additional dummy variable controlling for repeated movement.

Results of Model 1 show that movers do not differ significantly from non-movers in the premove period. In the post-move period movers receive on average more citations per patent compared to non-movers. The difference is significant at the $10 \%$ level. Factoring the multiple mover dummy into the regression (Model 2) decreases the effect of a move in the pre- and in the post-move period. This is not surprising, since the mover dummy captures part of the explanatory power of the multiple mover dummy in Models 1 and 3. However, the treatment group is still able to profit from a move. Whereas, in the pre-move period, movers 
showed a lower performance compared to non-movers, movers are able to catch-up with their non-moving control group in the after-move period. In particular, after the move took place, results do no longer show a significant difference between the treatment and the control inventors. The same results are obtained from the quantile regression, although only for the 0.75 quantile. The lower quantiles $(0.5$ and 0.25$)$ do not differ significantly across the four groups (mobile_pre, mobile_post, stay_pre, and stay_post). Again, mobile inventors are able to overtake the control group in the after-move period (Model 3c). If the multiple mover dummy is included (Model 4c) we observe a leveling of the treatment and the control group. However, movers are not ahead of non-movers in the pre-move period. Therefore, the endogeneity proposition of high inventive performance in the pre-move period increasing mobility which in turn increases inventive performance in the post-move period does not seem to hold. On the contrary, it appears that it is the "poor matches" that move to increase the match and also inventive performance. This in turn confirms the findings of Topel and Ward (1992). However, results reveal that higher quantiles, i.e. high performing inventors, seem to be better able to profit from a move and to catch-up with or to overtake the nonmovers.

At first glance, it is striking that that non-moving inventors receive less citations per patent in the post-move period compared to the pre-move period (Model 1: stay_pre dummy $=0.18$ ); the effect is significant at the $5 \%$ level. This outcome is especially surprising since the number of citations per patent rather increased over time. A plausible explanation for this outcome is the fact that inventors over time spend a larger share of their working hours on administrative duties, e.g., in case inventors get promoted into management positions (Hoisl 2007b). Consequently, these inventors become more and more invisible in terms of patents and also of citations. In part this effect is captured by the age variable. In particular, results show that age has a negative effect on the number of citations per patent and this effect 
increases at higher quantiles. However, to some extent the effect - inventors become less visible in terms of patents - is also measured by the stay_pre variable. Whereas the effect is negative and not significant at the 0.25 quantile it becomes positive (but still insignificant) at the 0.5 quantile. Finally, the effect increases and becomes highly significant at the 0.75 quantile (0.35). Since rather high performing inventors are promoted into management positions, it is not surprising that the negative coefficient of age and the positive coefficient of stay_pre increase at higher quantiles. ${ }^{10}$

Model 2 (OLS) and Model 4 (quantile regression) confirm that multiple movers produce patent applications that are on average more highly cited. Model 2 reveals that multiple movers receive on average 0.3 more citations. Descriptive statistics (Table 1) provide evidence that the mean number of citations per patent amounts to 3.3. Therefore, an increase by 0.3 means that the number of citations increases by $9 \%$. Consequently, the patent applications of multiple movers are more valuable compared to single movers or non-movers. Three interpretations of the multiple mover dummy seem possible: first, the dummy could represent experience effects. Possibly multiple movers may due to their "move-experience" be able to settle in or to adjust to a new environment faster. In addition, experienced movers may be capable of making better use of the knowledge learned from their new colleagues.

10 As a robustness check, the performance of the inventor before and after a selected move relative to a control group was analyzed using a difference-in-differences (DiD) approach. To measure inventive performance quantity measures (number of patents per inventor) as well as quality measures (grant rate, rate of withdrawal, rate of refusal, opposition rate, and number of citations) were employed. Results show that the number of applications does not change due to the move. However, the incident of a move has a positive impact on the mean share of applications granted. Furthermore, a move seems to have no impact on the share of patents refused by the patent examiner. In case the share of patent applications withdrawn by the applicant is considered, a move has a negative impact. Whereas the share of withdrawals prior to the move is larger for the movers than for the control group, it becomes smaller afterwards. The share of oppositions received within the opposition term of nine months after the patent was granted is lower in the movers' group before the move took place and higher afterwards. According to Harhoff and Hall (2003), the number of oppositions a patent received is a proxy for the value of the patent. Opposition results hence confirm that patent applications of the mobile inventors become more important after the move. In addition, citation counts also underline the proposition that a move has a positive effect on the value of after-move patent applications. The difference-in-differences estimator indicates that the number of citations increased after the move. The latter finding is consistent with the results described in this paper. 
These results point to the importance of an absorptive capacity at the individual inventor level (Cohen/Levinthal 1990). Second, inventors who move repeatedly may be different from single movers or non-movers with regard to their personal characteristics. For instance, multiple movers may be more flexible, more cosmopolitan, or more ambitious compared to the reference group (single and non-movers). These characteristics can again help multiple movers to settle in faster and consequently to increase inventive performance.

Finally, this outcome may also be explained by the fact that an inventor who moved works within the same technical area at his new job. Two R\&D teams working in the same area produce patent applications which form potential state-of-the-art to be referenced by the patent examiner of the EPO during the search process. The references from the search reports are used to calculate the number of citations from subsequent patents, therefore, more references also lead to more citations. To confirm this assumption, the citing patents have to be analyzed more closely. In particular one would have to find out whether part of the citations received by the after-move patents derive from the former employer of the mobile inventors. The former employer could be seen as a second source of self-citations.

Comparing the coefficients of the multiple mover dummy across different quantiles reveals that at the 0.25 quantile (Model $4 \mathrm{a}$ ) the coefficient of the multiple mover dummy is much smaller than at the 0.5 and the 0.75 quantiles (Models $4 \mathrm{~b}$ and 4c). Additionally, at the 0.25 quantile the effect is not significant at the $10 \%$ level. This means that multiple movers at the 0.25 quantile do not perform better compared to non- or single movers. A possible explanation is that low performing inventors are forced to change their job repeatedly, for instance, due to a dismissal. 
Table 2

Comparison of OLS and jointly estimated quantile regression

(dependent variable: average number of citations per patent for a 4 year window before and after the move) $(\mathrm{N}=1,408)$

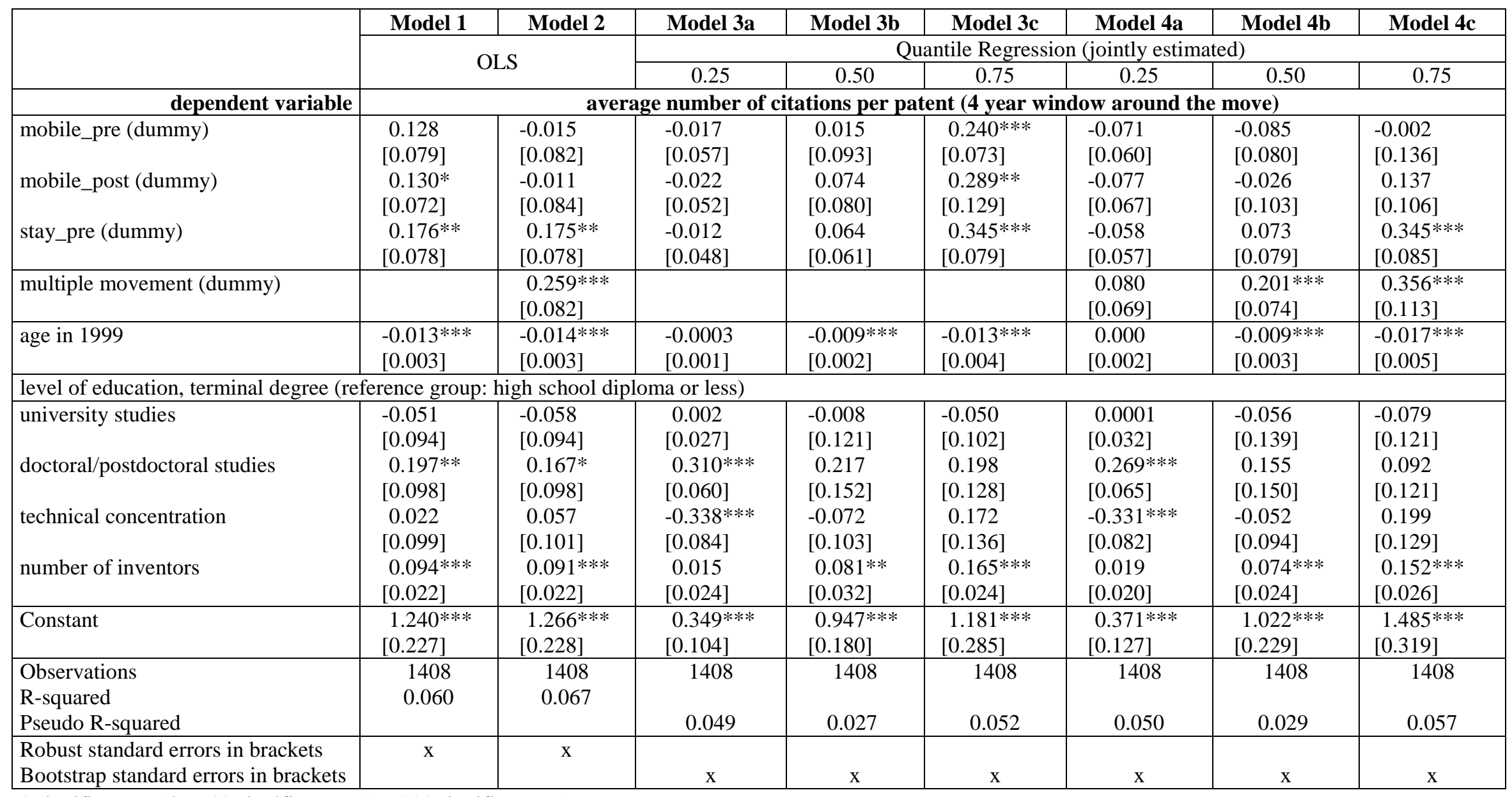

* significant at $10 \%$; $*$ significant at $5 \%$; *** significant at $1 \%$ 
The results given in Table 2 further reveal that the determinants of inventive performance also vary considerably across different points of the conditional performance distribution. Therefore, it can be assumed that the results of the quantile regression approach provide a better understanding of the factors influencing inventive performance. Whereas, at the 0.25 quantile, doctoral and postdoctoral studies increase the average number of citations per patent by $31 \%$ (Model 3a), a doctoral or a postdoctoral degree does not have a significant effect at the higher quantiles.

The same applies to the technical concentration. At the 25 quantile, higher specialized inventors produce less cited patents (Model 3a). At the 0.75 quantile technical concentration affects the dependent variable positively, although not significantly. Therefore, it seems that whereas a specialization on a small number of technical areas is a disadvantage at the bottom of the performance distribution, it may be an advantage at a higher performance level.

Additionally, results of Model 3 show that team size, which forms a proxy for firm size and the allocation of resources, does not matter at the bottom of the inventive performance distribution (0.25 quantile), but increases inventive performance at the higher quantiles. Additionally, the size of the effect at the 0.75 quantile is twice as high as that at the 0.5 quantile. At the 0.5 quantile, one more inventor per team leads to an increase of $2.4 \%$ in the average number of citations per patent. The increase in the average number of citations per patent at the 0.75 quantile amounts to $4.8 \%$. Obviously, high performing inventors are better able to make use of the resources they have at their disposal.

\section{Conclusion}

The objective of this study was to achieve a better understanding of the impact of a move on inventive performance. To reach this goal, a pre-post design with control group was employed to analyze the output of a group of mobile inventors relative to a non-mobile control group 
before and after a selected move. Additionally, a jointly estimated quantile regression approach was used to analyze whether returns from moving vary across different points of the conditional performance distribution of the inventors.

Data reveal that there are some striking gains from moving; in particular, it seems that "poor matches" move to improve match quality. Additionally, inventors at the upper end of the performance distribution are better able to benefit from a move to draw level with or to overtake their non-moving control inventors. However, not only the gains from moving but also the determinants of inventive performance vary across different points of the performance distribution. Finally, multiple movers turned out to perform better than single movers or non-movers. It is important to mention that the multiple mover coefficient cannot be interpreted with respect to a particular move, since the effect corresponds to the entire time period under consideration. Consequently, it is also possible that multiple movers are "star inventors" who were initially more productive. Therefore, future research needs to analyze multiple inventors more closely. In particular, surveys should try to identify star inventors and to analyze the effects of multiple movements on both groups (star inventors and average inventors) over time to find out whether repeated moving actually increases inventive performance or whether key inventors change their employers more often.

For R\&D management the importance of match quality for inventive performance implies that it is crucial to analyze the inventors' motives for moving in order to benefit from hiring a new inventor. Possible benefits from moving for inventors may be knowledge spillovers from new colleagues, monetary incentives, advancement, or new areas of application for existing knowledge. This information can be used to achieve a good match between the inventor and the hiring firm. Given a high match quality, it seems possible to not only transfer the knowledge of a high performing inventor but also to convert this knowledge into valuable inventions. 


\section{References}

Agrawal, A.K. / Cockburn, I.M. / McHale, J. (2006). Gone But Not Forgotten: Labor Flows, Knowledge Spillovers, and Enduring Social Capital, Journal of Economic Geography 6(5): 571-591.

Almeida, P. / Kogut, B. (1999). Localization of Knowledge and the Mobility of Engineers in Regional Networks, Management Science 45 (7): 905-917.

Arrow, K.J. (1962). Economic welfare and the allocation of resources for invention, in: R.R. Nelson (ed.), The Rate and Direction of Inventive Activity: Economic and Social Factors, Vol. 13 of NBER Special Conference Series, New Jersey: 609-625.

Buchinsky, M. (1994). Changes in U.S. Wage Structure 1963-1987: An Application of Quantile Regression, Econometrica 62(2): 405-458.

Chamberlain, G. (1994). Quantile Regression, Censoring and the Structure of Wage, in: Sims, C. (ed.), Advances in Econometrics, New York: 171-209.

Cohen, W.M. / Levinthal, D.A. (1990). Absorptive Capacity: A New Perspective on Learning and Innovation, Administrative Science Quarterly 35: 128-152.

Dosi, G. (1988). Technological Change and Economic Theory. Printer Publishers, New York.

Eide, E.R. / Showalter, M.H. (1999). Factors Affecting the Transmission of Earnings across Generations: A Quantile Regression Approach, The Journal of Human Resources 34(2): 253-267.

Fitzenberger, B. (1999). Wages and Employment Across Skill Groups, Heidelberg.

Gilfillan, S.C. (1935). The Sociology of Invention, Chicago.

Giuri P. / Mariani M. / Brusoni S. / Crespi G. / Francoz D. / Gambardella A. / Garcia-Fontes W. / Geuna A. / Gonzales R. / Harhoff D. / Hoisl K. / Lebas C. / Luzzi A. / Magazzini L. / Nesta L. / Nomaler O. / Palomeras N. / Patel P. / Romanelli M. / B. Verspagen (2007). Inventors and Invention Processes. Results from the PatVal-EU Survey. Research Policy, forthcoming.

Hahn, J. (1995). Bootstrapping Quantile Regression Estimators, Econometric Theory 11(1): 105-121.

Hall, B.H. (2004). Exploring the Patent Explosion, UC Berkeley und NBER Discussion Paper, Berkeley.

Harhoff, D. (2006). Patent Quantity and Quality: Trends and Policy Implications, in: B. Kahin, / D. Foray (eds.), Advancing Knowledge and the Knowledge Economy, Boston, MIT Press: 331-350. 
Harhoff, D. / Hall, B.H. (2003). Intellectual Property Strategy in the Global Cosmetics Industry, unpublished manuscript, University of Munich.

Harhoff, D. / Scherer, F.M. / Vopel, K. (2003). Citations, Family Size, Opposition and the Value of Patent Rights, Research Policy 32: 1343-1363.

Hoisl, K. (2007a). Tracing Mobile Inventors - The Causality between Inventor Mobility and Inventor Productivity, Research Policy 36(5): 619-636.

Hoisl, K. (2007b). A Closer Look at Inventive Output - The Role of Age and Career Paths, Discussion Paper.

Koenker, R. / Bassett, G. (1978). Regression Quantiles, Econometrica 46(1): 33-50.

Koenker, R. / Hallock, K.F. (2001). Quantile Regression, The Journal of Economic Perspectives 15(4): 143-156.

Moen, J. (2005). Is Mobility of Technical Personnel a Source of R\&D Spillovers?, Journal of Labor Economics 23 (1): 81-114.

OECD (1994). Using Patent Data as Science and Technological Indicators - Patent Manual 1994, Paris.

Polanyi, M. (1958). Personal Knowledge: Toward a post-critical Philosophy, Chicago.

Rosenkopf, L. / Almeida, P. (2003). Overcoming Local Search Through Alliances and Mobility, Management Science 49 (6): 751-766.

Schankerman, M. / Shalem, R. / Trajtenberg, M. (2006). Software Patents, Inventors and Mobility, Working Paper, August 2006.

Song, J. / Almeida, P. / Wu, G. (2003). Learning-by-Hiring: When is Mobility More Likely to Facilitate Interfirm Knowledge Transfer?, Management Science 49(4): 351-365.

Topel, R.H. / Ward, P.W. (1992). Job Mobility and the Careers of Young Men, The Quarterly Journal of Economics 107(2): 439-479.

Trajtenberg, M. (2005). Recombinant Ideas: The Mobility of Inventors and the Productivity of Research, CEPR-Conference, Munich, May 26-28, 2005.

Trajtenberg, M. / Shiff, G. / Melamed, R. (2006). The "Names Game“: Harnessing Inventors' Patent Data for Economic Research, NBER WP No. 12479, Cambridge, Mass. 